\title{
A HYBRID EXPLICIT-IMPLICIT SCHEME FOR THE TIME-DEPENDENT WIGNER EQUATION*
}

\author{
Haiyan Jiang \\ Department of Mathematics and Statistics,Beijing Institute of Technology, Beijing 100081, China \\ Email: hyjiang@bit.edu.cn \\ Tiao $\mathrm{Lu}^{1)}$ \\ CAPT, HEDPS, LMAM, IFSA Collaborative Innovation Center of MoE, \\ School of Mathematical Sciences, Peking University, Beijing 100871, China \\ Email: tlu@math.pku.edu.cn \\ $\mathrm{Xu}$ Yin \\ Department of Mathematics and Statistics,Beijing Institute of Technology, Beijing 100081, China \\ Email:2120161343@bit.edu.cn
}

\begin{abstract}
This paper designs a hybrid scheme based on finite difference methods and a spectral method for the time-dependent Wigner equation, and gives the error analysis for the full discretization of its initial value problem. An explicit-implicit time-splitting scheme is used for time integration and the second-order upwind finite difference scheme is used to discretize the advection term. The consistence error and the stability of the full discretization are analyzed. A Fourier spectral method is used to approximate the pseudo-differential operator term and the corresponding error is studied in detail. The final convergence result shows clearly how the regularity of the solution affects the convergence order of the proposed scheme. Numerical results are presented for confirming the sharpness of the analysis. The scattering effects of a Gaussian wave packet tunneling through a Gaussian potential barrier are investigated. The evolution of the density function shows that a larger portion of the wave is reflected when the height and the width of the barrier increase.
\end{abstract}

Mathematics subject classification: 65M06, 65M70.

Key words: Finite difference method, Spectral method, Hybrid scheme, Error analysis, Wigner equation.

\section{Introduction}

With the development of micro-manufacturing engineering and technology, the quantum effects (such as the tunnelling effects in Resonant Tunnelling Diodes) play an important role in transport property and can not be neglected. In order to clearly understand the quantum effects, quantum transport models have to be considered. One of such models is the Wigner equation, which was proposed by Wigner in 1932 as a quantum correction of the classical statistical mechanics in the phase space [1]. As its classical counterparts, the Boltzmann equation and the Vlasov equation are popular models in simulating of carrier transport in classical mechanics for clear description of scattering effects and boundary conditions. Thus, the Wigner equation has advantages over other quantum frameworks in handling scattering mechanisms and boundary

\footnotetext{
* Received May 1, 2018 / Accepted June 27, 2019 /

Published online August 16, 2019 /

1) Corresponding author
} 
conditions [2]. The Wigner equation is also suitable for describing time dependent dynamics and connecting quantum and semi-classical regimes [3], and it has been found many applications in different fields, e.g., quantum optics [4], nano-scale semiconductor devices [5].

The Wigner equation and its numerical solution have attracted more and more attention since Frensley successfully reproduced the negative differential resistance phenomenon of resonant tunnelling diodes (RTDs) in [5], where the first-order upwind finite difference method was used. Then the second-order upwind finite difference method was proposed and used to study the tunneling characteristics of double-barrier semiconductor structures [6,7]. Many more numerical methods have developed for the Wigner equation, e.g., a WENO solver [8], a spectral method [9], a spectral collocation method [10], high-order upwind finite-difference methods [11], a Gaussian beam method [12], moment methods [13,14] and a cell average spectral element method [15]. Many mathematicians also devoted to analyze the Wigner equation. Markowich proved the existence and uniqueness of the solution to the initial-value problem of the time-dependent Wigner equation by studying the equivalence between the quantum Liouville equation and the Schrödinger equation [16]. Jiang et al. studied the effect of the inflow boundary conditions of the stationary Wigner equation in [17,18], and proposed a device adaptive inflow boundary condition in [19]. Readers interested in the research on the well-posedness of related problems can refer to, e.g., [16, 20-23].

There are also many works on the Wigner equation that focus on the numerical simulation and investigate the parallel computing, where the nonlinear iteration technique is used when the self-consistent potential is included. However, works on a detailed numerical analysis of the consistency, convergence and stability of numerical methods for the Wigner equation is still inadequate. In this paper, we propose a hybrid explicit-implicit scheme of finite difference methods and a Fourier spectral method for the time-dependent Wigner equation. We adopt the second-order upwind finite difference method for the discretization of the advection term as did in [6], which has gained its popularity comparing with the first-order upwind finite difference method used in [5]. The pseudo-differential term of the Wigner equation, which corresponds to the force field, is approximated by the Fourier spectral method. Because of the non-locality of the pseudo-differential operator of the Wigner equation, the semi-discretization in the phase space results a matrix with much more nonzero elements than that of the Vlasov equation. Thus the forward Euler method used in [5] is popular since a linear system (whose matrix is not very sparse) need not to be solved at every time step. However, the time step in the forward Euler method is restricted due to the CFL condition restraint. An implicit scheme can be used to alleviate the CFL restraint. For example, a backward Euler method is used in [9]. In order to take advantage of the computational efficiency of the explicit method and the big time step of the implicit method, we apply the implicit method for the advection term (which actually can be solved very efficiently) and the explicit method for the pseudo-differential operator term. A prediction-correction technique is implemented to achieve a second-order accuracy in time integration. For the discretization with respect to velocity, many methods have proposed, such as the collocation spectral method [9], the adaptive cell-average spectral element method [15], the moment method [13,14]. In this paper, we adopt the collocation spectral method to approximate the pseudo-differential operator term as used in [9], which guarantees the conservative conditions automatically. The high-order truncation error of the proposed full discretization scheme is proved, and the stability is analyzed by using the von Neumann method. Finally, we give a proof of the convergence of the full discretization scheme.

This paper is arranged as following: Firstly, we introduce an initial boundary value prob- 
lem for the time-dependent Wigner equation in a finite phase space. Secondly, the hybrid explicit-implicit (EI) scheme is developed to solve the time-dependent Wigner equation. Thirdly, the local truncation error, the stability and the convergence of the high-order EI scheme are analyzed. Finally, numerical simulations are implemented to validate the convergence of the scheme, and the scheme is adopted to simulate the evolution of Gaussian wave packet tunneling through a Gaussian barrier.

\section{The Wigner Equation}

The initial value problem of the Wigner equation

$$
\left\{\begin{array}{l}
\Re_{0} f(x, k, t):=\frac{\partial f(x, k, t)}{\partial t}+\frac{k \hbar}{m} \frac{\partial f(x, k, t)}{\partial x}+\frac{1}{2 \pi \hbar} \Theta(f)(x, k, t)=0, \\
(x, k) \in(-\infty, \infty) \times(-\infty, \infty), \quad t>0 \\
f(x, k, 0)=f_{0}(x, k),
\end{array}\right.
$$

describes how the Wigner distribution function $f(x, k, t)$ evolves in the phase space $(x, k)$ with time $t$ when the initial distribution $f_{0}(x, k)$ is given. Here $m$ is the effective mass, $\hbar$ the reduced Plank constant, and $\Theta$ is the convolution operator

$$
\left\{\begin{array}{l}
\Theta(f)(x, k, t)=\int_{-\infty}^{\infty} V_{w}\left(x, k-k^{\prime}\right) f\left(x, k^{\prime}, t\right) \mathrm{d} k^{\prime} \\
V_{w}(x, k)=\mathrm{i} \int_{-\infty}^{\infty}\left[V\left(x+\frac{r}{2}\right)-V\left(x-\frac{r}{2}\right)\right] \exp (-\mathrm{i} k r) \mathrm{d} r
\end{array}\right.
$$

where $V(x)$ is the potential energy function to denote the external barrier imposed to cause the scattering of the wave, and then changes the distribution function. In our case, $V(x)$ is assumed to be independent of time. $\Theta(f)$ is a pseudo-differential operator term for having differential form if $V(x)$ is infinitely differentiable. The Wigner equation is a kinetic equation in the phase space which includes quantum effects, so it is also called quantum Boltzmann equation. Inflow boundary condition is usually imposed on Wigner equation due to its similarity with the Boltzmann equation. The initial value problem (2.1) can be recast as the following initial inflow boundary value problem (IBVP) in a finite domain

$$
\begin{cases}\Re_{0} f(x, k, t)=0, & -\frac{L}{2}<x<\frac{L}{2}, t>0, \\ f(x, k, t)=f_{L}(k), & x \leq-\frac{L}{2}, k>0, t>0, \\ f(x, k, t)=f_{R}(k), & x \geq \frac{L}{2}, k<0, t>0, \\ f(x, k, 0)=f_{0}(x, k), & -\frac{L}{2}<x<\frac{L}{2},\end{cases}
$$

where $L$ is the length of the device which is sandwiched by two contacts.

\section{The Hybrid Explicit-Implicit Scheme}

Usually it is impossible to solve the initial value problem (2.1) or the IBVP (2.3) analytically for the complexity of pseudo-differential operator term. The finite difference method and Fourier 
spectral method are used to solve the IBVP (2.3) numerically. The derivatives with respect to time $t$ and space $x$ are approximated by applying the finite difference method. The Fourier spectral method is applied to approximate the double integral in the pseudo-differential operator term $\Theta(f)$. A high-order hybrid accurate scheme is designed by the following three steps.

\subsection{The discretization of pseudo-differential operator term}

Firstly, we consider the discretization of $\Theta(f)$ which is composed of a double integral with respect to the variables $k$ and $r$. The Fourier spectral methods are used for the semi-discrete Wigner equation in the $k$ direction. Denoting $L_{k}$ as the length of the computation domain for the wave number $k$, Wigner function $f(x, k, t)$ can be expanded as the Fourier series with respect to $k$

$$
\begin{aligned}
& f(x, k, t)=\sum_{n=-\infty}^{+\infty} \rho_{n}(x, t) \exp \left(-\mathrm{i} \frac{2 n \pi}{L_{k}} k\right), \\
& \rho_{n}(x, t)=\frac{1}{L_{k}} \int_{-\frac{L_{k}}{2}}^{\frac{L_{k}}{2}} f(x, k, t) \exp \left(\mathrm{i} \frac{2 n \pi}{L_{k}} k\right) \mathrm{d} k,
\end{aligned}
$$

where $\rho_{n}(x, t)$ are the Fourier coefficients. Substituting the Fourier series (3.1) into $\Theta(f)$ and exchanging the integration and summation yield

$$
\begin{aligned}
\Theta(f)(x, k, t) & =\int_{-\infty}^{\infty} V_{w}\left(x, k-k^{\prime}\right) \sum_{n=-\infty}^{+\infty} \rho_{n}(x, t) \exp \left(-\mathrm{i} \frac{2 n \pi}{L_{k}} k^{\prime}\right) \mathrm{d} k^{\prime} \\
& =2 \pi \mathrm{i} \sum_{n=-\infty}^{+\infty} \rho_{n}(x, t)\left[V\left(x+\frac{n \pi}{L_{k}}\right)-V\left(x-\frac{n \pi}{L_{k}}\right)\right] \exp \left(-\mathrm{i} \frac{2 n \pi}{L_{k}} k\right) .
\end{aligned}
$$

If the Fourier series is cut off as

$$
f^{N}(x, k, t)=\sum_{n=-N}^{N-1} \rho_{n}(x, t) \exp \left(-\mathrm{i} \frac{2 n \pi}{L_{k}} k\right)
$$

applying operator $\Theta$ to $f^{N}$

$$
\Theta\left(f^{N}\right)(x, k, t)=2 \pi \mathrm{i} \sum_{n=-N}^{N-1} \rho_{n}(x, t)\left[V\left(x+\frac{n \pi}{L_{k}}\right)-V\left(x-\frac{n \pi}{L_{k}}\right)\right] \exp \left(-\mathrm{i} \frac{2 n \pi}{L_{k}} k\right) .
$$

The Fourier coefficients $\rho_{n}(x, t)$ will be approximated by the discrete Fourier transform(DFT) coefficients

$$
\bar{\rho}_{n}(x, t)=\frac{h_{k}}{L_{k}} \sum_{j=-N}^{N-1} f\left(x, k_{j}, t\right) \exp \left(\mathrm{i} \frac{2 n \pi}{L_{k}} k_{j}\right),
$$

where

$$
k_{j}=\left(j+\frac{1}{2}\right) h_{k}, \quad j=-N,-N+1, \cdots, N-1,
$$

are the sampling points. They are obtained by partitioning interval $\left[-\frac{L_{k}}{2}, \frac{L_{k}}{2}\right]$ into a uniform mesh with mesh size $h_{k}=\frac{L_{k}}{2 N}$. The fast Fourier transform (FFT) is used to calculate these DFT coefficients. The full discretization formulation of the pseudo-differential operator term is

$$
\widetilde{\Theta}(f)(x, k, t)=2 \pi \mathrm{i} \sum_{n=-N}^{N-1} \bar{\rho}_{n}(x, t)\left[V\left(x+\frac{n \pi}{L_{k}}\right)-V\left(x-\frac{n \pi}{L_{k}}\right)\right] \exp \left(-\mathrm{i} \frac{2 n \pi}{L_{k}} k\right) .
$$


Noting that $\left[V\left(x+\frac{r}{2}\right)-V\left(x-\frac{r}{2}\right)\right]$ is odd with respect to $r$, the calculation formula for $\Theta(f)$ can be simplified as

$$
\left\{\begin{array}{l}
\widetilde{\Theta}(f)(x, k, t)=h_{k} \sum_{j^{\prime}=-N}^{N-1} \widetilde{V}_{w}\left(x, k-k_{j^{\prime}}\right) f\left(x, k_{j^{\prime}}, t\right), \\
\widetilde{V}_{w}(x, k)=2 h_{r} \sum_{n=0}^{N-1}\left[V\left(x+\frac{n h_{r}}{2}\right)-V\left(x-\frac{n h_{r}}{2}\right)\right] \sin \left(n h_{r} k\right),
\end{array}\right.
$$

where $h_{r}=\frac{2 \pi}{L_{k}}$. Denote $L_{r}=2 N h_{r}=\frac{2 \pi}{h_{k}}$, where $L_{r}$ is the coherence length. In fact, these are the conservative conditions for quadrature rules in [17,18]. It is concluded that the Fourier spectral methods satisfy the conservative condition naturally. After the semi-discretization in the $k$ direction, the Wigner equation (2.3) is approximated by a hyperbolic system

$$
\begin{gathered}
\Re_{1} f\left(x, k_{j}, t\right):=\frac{\partial f\left(x, k_{j}, t\right)}{\partial t}+\frac{\hbar}{m} k_{j} \frac{\partial f\left(x, k_{j}, t\right)}{\partial x}+\frac{1}{2 \pi \hbar} \widetilde{\Theta}(f)\left(x, k_{j}, t\right)=0, \\
j=-N,-N+1, \cdots, N-1 .
\end{gathered}
$$

Defining a vector function $F(x, t)$ as

$$
F(x, t)=\left[f\left(x, k_{-N}, t\right), f\left(x, k_{-N+1}, t\right), \cdots, f\left(x, k_{N-1}, t\right)\right]^{\mathrm{T}},
$$

where $\mathrm{T}$ means the transpose of a matrix, the matrix form of the hyperbolic system (3.9) can be written as

$$
\frac{\partial F(x, t)}{\partial t}+K \frac{\partial F(x, t)}{\partial x}+A(x) F(x, t)=0,
$$

where $K$ is a diagonal matrix, $K=\frac{\hbar}{m} \operatorname{diag}\left(k_{-N}, k_{-N+1}, \cdots, k_{N-1}\right)$, and

$$
A(x)=\left(a_{j j^{\prime}}(x)\right)_{2 N \times 2 N}, \quad a_{j j^{\prime}}(x)=\frac{h_{k}}{2 \pi \hbar}\left(\widetilde{V}_{w}\left(x, k_{j}-k_{j^{\prime}}\right)\right),
$$

for all $j, j^{\prime}=-N,-N+1, \cdots, N-1$. The discretization formulation of the pseudo-differential operator term can also be written as

$$
\frac{1}{2 \pi \hbar} \widetilde{\Theta}(f)\left(x, k_{j}, t\right)=\bar{a}_{j}(x) F(x, t),
$$

where $\bar{a}_{j}(x)$ is the $j$-th line of matrix $A(x)$.

\subsection{The discretization in the $x$ direction}

The second-order upwind finite difference scheme is used to discretize the advection term in (3.9). $\left[-\frac{L}{2}, \frac{L}{2}\right]$ is partitioned into $N_{x}$ equal sized subintervals, and grid points are

$$
x_{l}=-\frac{L}{2}+l h_{x}, \quad h_{x}=\frac{L}{N_{x}}, \quad l=0,1,2, \cdots, N_{x} .
$$

We define the difference operator $D^{ \pm}$, which is the second-order accurate one-side difference scheme of the first-order derivative with respect to $x$

$$
\begin{aligned}
& D^{+} g\left(x_{l}\right)=\frac{g\left(x_{l-2}\right)-4 g\left(x_{l-1}\right)+3 g\left(x_{l}\right)}{2 h_{x}}, \\
& D^{-} g\left(x_{l}\right)=\frac{-g\left(x_{l+2}\right)+4 g\left(x_{l+1}\right)-3 g\left(x_{l}\right)}{2 h_{x}},
\end{aligned}
$$


where $g\left(x_{l}\right)$ is the value of function $g(x)$ at $x_{l}$. Denoting

$$
D_{j}= \begin{cases}D^{+} & k_{j}>0 \\ D^{-} & k_{j}<0\end{cases}
$$

and applying (3.13) to approximate the first-order derivative with respect to $x$, the discretization of (3.9) yields

$$
\begin{aligned}
\Re_{2} f_{l j}(t):= & \frac{\mathrm{d} f_{l j}(t)}{\mathrm{d} t}+\frac{\hbar}{m} k_{j} D_{j} f_{l j}(t)+\bar{a}_{j}\left(x_{l}\right) F_{l}(t)=0, \\
& l=1, \cdots, N_{x}-1, \quad j=-N,-N+1, \cdots, N-1,
\end{aligned}
$$

where $f_{l j}(t)$ is the approximate value of $f(x, k, t)$ at $\left(x_{l}, k_{j}\right)$ and the corresponding vector function is $F_{l}(t)=\left[f_{l,-N}(t), f_{l,-N+1}(t), \ldots, f_{l, N-1}(t)\right]^{\mathrm{T}}$. Denoting $D$ as the diagonal operator matrix $D=\operatorname{diag}\left(D_{-N}, D_{-N+1}, \cdots, D_{N-1}\right)$, the matrix form of (3.14) can be written as

$$
\frac{\mathrm{d} F_{l}(t)}{\mathrm{d} t}+K D F_{l}(t)+A\left(x_{l}\right) F_{l}(t)=0, \quad l=1, \cdots, N_{x}-1 .
$$

In fact, $(3.15)$ is the discretization of $(3.10)$ in the $x$ direction.

\subsection{The discretization in the $t$ direction}

After the discretizations in the $k$ and $x$ directions, a first-order ordinary differential system (3.14) which only depends on $t$ is obtained. By combining the computational efficiency of the explicit scheme and robust stability of the implicit scheme, a prediction-correction scheme is proposed to discretize (3.14) in the $t$ direction

$$
\left\{\begin{array}{l}
\frac{\bar{f}_{l j}^{n+1}-f_{l j}^{n}}{h_{t}}+\frac{\hbar}{m} k_{j} D_{j}\left(\frac{f_{l j}^{n}+\bar{f}_{l j}^{n+1}}{2}\right)+\bar{a}_{j}\left(x_{l}\right) F_{l}^{n}=0 \\
\frac{f_{l j}^{n+1}-f_{l j}^{n}}{h_{t}}+\frac{\hbar}{m} k_{j} D_{j}\left(\frac{f_{l j}^{n}+f_{l j}^{n+1}}{2}\right)+\bar{a}_{j}\left(x_{l}\right)\left(\frac{\bar{F}_{l}^{n+1}+F_{l}^{n}}{2}\right)=0
\end{array}\right.
$$

where $f_{l j}^{n}$ is the approximate value of $f_{l j}(t)$ at $t_{n}$ and the vector $F_{l}^{n}=\left[f_{l,-N}^{n}, f_{l,-N+1}^{n}, \cdots\right.$, $\left.f_{l, N-1}^{n}\right]^{\mathrm{T}}$. Although the scheme (3.16) includes implicit character in upwind term and convolution integration term, $\bar{f}_{l j}^{n}$ and $f_{l j}^{n}$ can be calculated explicitly for inflow boundary conditions. The prediction step determines the relation of the prediction value and the initial value

$$
\bar{F}_{l}^{n+1}=T F_{l}^{n}:=\left(I+\frac{h_{t}}{2} K D\right)^{-1}\left(I-\frac{h_{t}}{2} K D+A_{l}\right) F_{l}^{n} .
$$

While $\bar{f}_{l j}^{n+1}$ is calculated by the prediction step and substituted into the correction step, the relation of $f_{l j}^{n+1}$ and $f_{l j}^{n}$ is derived

$$
\Re_{3}\left(f_{l j}^{n+1}, f_{l j}^{n}\right):=\frac{f_{l j}^{n+1}-f_{l j}^{n}}{h_{t}}+\frac{\hbar}{m} k_{j} D_{j}\left(\frac{f_{l j}^{n}+f_{l j}^{n+1}}{2}\right)+\bar{a}_{j}\left(x_{l}\right)\left(\frac{T+I}{2}\right) F_{l}^{n}=0 .
$$

So far, the full discretization of the time dependent Wigner equation is obtained. We name the scheme (3.16) as explicit-implicit (EI) scheme. The high-order accuracy and the unconditional stability of the scheme will be analyzed in Section 4 . 


\section{Error Analysis}

In this section, the proof of the high-order accuracy of the EI scheme will be given. The stability and convergence of the EI scheme are also analyzed. The main results in this section are Theorems 4.1-4.3.

\subsection{Truncation error}

The truncation error $\tau$ is the difference between the full discretization operator $\Re_{3}$ and Wigner operator $\Re_{0}$ applying to $f(x, k, t)$

$$
\tau\left(x_{l}, k_{j}, t_{n}\right)=\Re_{3}\left(f\left(x_{l}, k_{j}, t_{n+1}\right), f\left(x_{l}, k_{j}, t_{n}\right)\right)-\Re_{0} f\left(x_{l}, k_{j}, t_{n}\right) .
$$

It is convenient to separate the truncation error $\tau\left(x_{l}, k_{j}, t_{n}\right)$ into two parts. One part comes from the difference methods and the other is due to the Fourier spectral method. Introducing the following notations

$$
\begin{aligned}
& \tau_{1}\left(x_{l}, k_{j}, t_{n}\right):=\Re_{1} f\left(x_{l}, k_{j}, t_{n}\right)-\Re_{0} f\left(x_{l}, k_{j}, t_{n}\right)=\frac{1}{2 \pi \hbar}[\Theta(f)-\widetilde{\Theta}(f)]\left(x_{l}, k_{j}, t_{n}\right), \\
& \tau_{2}\left(x_{l}, k_{j}, t_{n}\right):=\Re_{3}\left(f\left(x_{l}, k_{j}, t_{n+1}\right), f\left(x_{l}, k_{j}, t_{n}\right)\right)-\Re_{1} f\left(x_{l}, k_{j}, t_{n}\right),
\end{aligned}
$$

it is obvious that,

$$
\tau\left(x_{l}, k_{j}, t_{n}\right)=\tau_{1}\left(x_{l}, k_{j}, t_{n}\right)+\tau_{2}\left(x_{l}, k_{j}, t_{n}\right) .
$$

Lemma 4.1. Assume that there exist constants $M$ and $Q$, such that

$$
\begin{aligned}
& |V(x)| \leq M, \\
& \left|\frac{\partial^{j} f(x, k, t)}{\partial k^{j}}\right| \leq Q,\left(|k|<\frac{L_{k}}{2}\right) \quad j=0,1, \cdots, p, \\
& \frac{\partial^{j} f(x, k, t)}{\partial k^{j}}=0 \quad\left(|k| \geq \frac{L_{k}}{2}\right), \quad j=0,1, \cdots, p-1,
\end{aligned}
$$

for all $x \in\left[-\frac{L}{2}, \frac{L}{2}\right], k \in\left[-\frac{L_{k}}{2}, \frac{L_{k}}{2}\right]$ and $t \geq 0$. Then the local truncation error $\tau_{1}\left(x_{l}, k_{j}, t_{n}\right)$ satisfies

$$
\left|\tau_{1}\left(x_{l}, k_{j}, t_{n}\right)\right| \leq \frac{M Q L_{k}}{2 \pi^{p+1} \hbar}\left(\frac{5 p-1}{p-1}\right) h_{k}^{p-1} .
$$

Proof. The truncation error $\tau_{1}$ caused by the discretization of the pseudo-differential operator term is

$$
\tau_{1}\left(x_{l}, k_{j}, t_{n}\right)=\frac{1}{2 \pi \hbar}[\Theta(f)-\widetilde{\Theta}(f)]\left(x_{l}, k_{j}, t_{n}\right) .
$$

The discretization of the pseudo-differential operator term consists of two steps. Firstly, the infinite Fourier series are truncated into a finite series $f^{N}(x, k, t)$. Secondly, the Fourier coefficients are calculated approximately by DFT. Thus the truncation error $\tau_{1}$ can be separated into two parts. One is the summation of the cut-off Fourier coefficients and the other is the total difference between the Fourier coefficients and discrete Fourier coefficients,

$$
|\Theta(f)-\widetilde{\Theta}(f)| \leq\left|\Theta(f)-\Theta\left(f^{N}\right)\right|+\left|\Theta\left(f^{N}\right)-\widetilde{\Theta}(f)\right| .
$$


Denoting $V_{d}(x, r)=V\left(x+\frac{r}{2}\right)-V\left(x-\frac{r}{2}\right)$ and assuming $|V(x)| \leq M$, the errors can be estimated according to (3.2), (3.4) and (3.7),

$$
\begin{aligned}
\left|\Theta(f)-\Theta\left(f^{N}\right)\right| & =\left|\left(\sum_{n=-\infty}^{-N-1}+\sum_{n=N}^{+\infty}\right) \rho_{n}(x, t) V_{d}\left(x, n h_{r}\right) \exp \left(\mathrm{i} n h_{r} k\right)\right| \\
& \leq 2 M\left(\sum_{n=-\infty}^{-N-1}+\sum_{n=N}^{+\infty}\right)\left|\rho_{n}(x, t)\right|, \\
\left|\Theta\left(f^{N}\right)-\widetilde{\Theta}(f)\right| & =\left|\sum_{n=-N}^{N-1}\left(\rho_{n}(x, t)-\bar{\rho}_{n}(x, t)\right) V_{d}\left(x, n h_{r}\right) \exp \left(\mathrm{i} n h_{r} k\right)\right| \\
& \leq 2 M \sum_{n=-N}^{N-1}\left|\rho_{n}(x, t)-\bar{\rho}_{n}(x, t)\right| .
\end{aligned}
$$

The relation of the discrete Fourier coefficients and Fourier coefficients is

$$
\bar{\rho}_{n}(x, t)=\rho_{n}(x, t)+\sum_{l \neq 0} \rho_{n+2 l N}(x, t) .
$$

Thus we have

$$
\sum_{n=-N}^{N-1}\left|\bar{\rho}_{n}(x, t)-\rho_{n}(x, t)\right|=\sum_{n=-N}^{N-1} \sum_{l \neq 0}\left|\rho_{n+2 l N}(x, t)\right| .
$$

It is obvious that

$$
\sum_{n=-N}^{N-1} \bar{\rho}_{n}(x, t)=\sum_{n=-\infty}^{\infty} \rho_{n}(x, t), \quad \sum_{n=-N}^{N-1}\left|\bar{\rho}_{n}(x, t)\right|<\sum_{n=-\infty}^{\infty}\left|\rho_{n}(x, t)\right| .
$$

The main task is to give the estimation of the Fourier coefficient. The Wigner function $f(x, k, t)$ and its $(p-1)$-th $(p>1)$ order derivative are assumed to be continuous. As a quantum distribution function, the Wigner function is damping very fast with $|k|$ increasing. Applying integration by parts $p$ times and using assumption (4.4), it can be attained

$$
\begin{aligned}
\rho_{n}(x, t) & =\frac{1}{L_{k}} \int_{-\frac{L_{k}}{2}}^{\frac{L_{k}}{2}} f(x, k, t) \exp \left(\mathrm{i} \frac{2 n \pi}{L_{k}} k\right) \mathrm{d} k \\
& =\frac{1}{L_{k}}\left(\frac{L_{k}}{\mathrm{i} 2 n \pi}\right)^{p} \int_{-\frac{L_{k}}{2}}^{\frac{L_{k}}{2}} \frac{\partial^{p} f(x, k, t)}{\partial k^{p}} \exp \left(\mathrm{i} \frac{2 n \pi}{L_{k}} k\right) \mathrm{d} k .
\end{aligned}
$$

Using (4.4) and (4.13), the following estimate arrives

$$
\left|\rho_{n}(x, t)\right|<Q\left(\frac{L_{k}}{2 \pi}\right)^{p} \frac{1}{|n|^{p}} \quad(n \neq 0) .
$$

Thus 1-norm of the discrete Fourier coefficients and Fourier coefficients are bounded by

$$
\sum_{n=-N}^{N-1}\left|\bar{\rho}_{n}(x, t)\right|<\sum_{n=-\infty}^{\infty}\left|\rho_{n}(x, t)\right|<Q\left(1+\left(\frac{L_{k}}{\pi}\right)^{2}\right) .
$$


It is easily derived that

$$
\sum_{n=N+1}^{\infty} \frac{1}{n^{p}}<\int_{0}^{\infty} \frac{1}{(N+x)^{p}} \mathrm{~d} x=\left(\frac{1}{p-1}\right) \frac{1}{N^{p-1}}
$$

Thus we can obtain the following estimates:

$$
\begin{aligned}
\left(\sum_{n=-\infty}^{-N-1}+\sum_{n=N}^{n=+\infty}\right) \frac{1}{|n|^{p}}<\frac{1}{N^{p}}+\sum_{n=N+1}^{\infty} \frac{2}{|n|^{p}}<\left(\frac{p+1}{p-1}\right) \frac{1}{N^{p-1}}, & \sum_{l=1}^{\infty} \frac{1}{(n+2 l N)^{p}}<\frac{1}{(n+2 N)^{p}}+\int_{1}^{\infty} \frac{1}{(n+2 N x)^{p}} \mathrm{~d} x \\
& <\frac{1}{N}\left(\frac{2 p-1}{2 p-2}\right) \frac{1}{(n+2 N)^{p-1}}, \\
\sum_{l=-\infty}^{-1} \frac{1}{|n+2 l N|^{p}} & =\sum_{l=1}^{\infty} \frac{1}{(2 l N-n)^{p}}<\frac{1}{N}\left(\frac{2 p-1}{2 p-2}\right) \frac{1}{(2 N-n)^{p-1}} .
\end{aligned}
$$

Then we can get

$$
\begin{aligned}
\sum_{n=-N}^{N-1} \sum_{l \neq 0} \frac{1}{|n+2 l N|^{p}} & <\sum_{n=-N}^{N-1} \frac{1}{N}\left(\frac{2 p-1}{2 p-2}\right)\left(\frac{1}{(n+2 N)^{p-1}}+\frac{1}{(2 N-n)^{p-1}}\right) \\
& <2\left(\frac{2 p-1}{p-1}\right) \frac{1}{N^{p-1}} .
\end{aligned}
$$

Using the conclusions above, we obtain the following estimate

$$
|\Theta(f)-\widetilde{\Theta}(f)| \leq 2 M Q_{1}\left(\frac{5 p-1}{p-1}\right) \frac{1}{N^{p-1}},
$$

where $Q_{1}=Q\left(\frac{L_{k}}{2 \pi}\right)^{p}$. According to (4.6), the conclusion (4.5) can be attained.

Lemma 4.2. Assume that there exist constants $M$ and $P$, such that

$$
\begin{aligned}
& |V(x)| \leq M, \\
& \left|\frac{\partial^{q} f(x, k, t)}{\partial x^{q}}\right|+\left|\frac{\partial^{3} f(x, k, t)}{\partial x^{q_{1}} \partial k^{q_{2}} \partial t^{q_{3}}}\right|+\left|\frac{\partial^{4} f(x, k, t)}{\partial x^{2} \partial k^{2}}\right|+\left|\frac{\partial^{4} f(x, k, t)}{\partial x \partial t \partial k^{2}}\right|<P, \\
& \quad\left(q_{1}+q_{2}+q_{2}=3,0 \leq q_{1}, q_{2}, q_{3} \leq 3 ; q=1,2\right),
\end{aligned}
$$

for all $x \in\left[-\frac{L}{2}, \frac{L}{2}\right], k \in\left[-\frac{L_{k}}{2}, \frac{L_{k}}{2}\right]$ and $t \geq 0$. Then the local truncation error $\tau_{2}\left(x_{l}, k_{j}, t_{n}\right)$ satisfies

$$
\left|\tau_{2}\right| \leq h_{t}^{2}\left(\frac{1}{6}+\frac{\hbar}{4 m}+\frac{28 M}{\hbar}\left(2+\frac{L_{k}}{2}\right)^{2}\left(1+\left(\frac{L_{k}}{\pi}\right)^{2}\right)\right) P+h_{x}^{2}\left(\frac{L_{k} \hbar}{2 m}\right) P,
$$


Proof. In order to analyze the truncation error due to the finite difference method, the Taylor expansions which will be used later in the discussion of the truncation error are listed

$$
\begin{aligned}
& \frac{f\left(x_{l}, k_{j}, t_{n+1}\right)-f\left(x_{l}, k_{j}, t_{n}\right)}{h_{t}} \\
= & \frac{\partial f\left(x_{l}, k_{j}, t_{n}\right)}{\partial t}+\frac{1}{2} \frac{\partial^{2} f\left(x_{l}, k_{j}, t_{n}\right)}{\partial t^{2}} h_{t}+\frac{1}{3 !} \frac{\partial^{3} f\left(x_{l}, k_{j}, t_{n}+\theta h_{t}\right)}{\partial t^{3}} h_{t}^{2}, \\
& D^{+} f\left(x_{l}, k_{j}, t_{n}\right) \\
= & \frac{\partial f\left(x_{l}, k_{j}, t_{n}\right)}{\partial x}+\frac{1}{3}\left[\frac{\partial^{3} f\left(x_{l}-\theta h_{x}, k_{j}, t_{n}\right)}{\partial x^{3}}-2 \frac{\partial^{3} f\left(x_{l}-2 \theta h_{x}, k_{j}, t_{n}\right)}{\partial x^{3}}\right] h_{x}^{2}, \\
& D^{+} f\left(x_{l}, k_{j}, t_{n+1}\right)=\frac{\partial f\left(x_{l}, k_{j}, t_{n}\right)}{\partial x}+\frac{\partial^{2} f\left(x_{l}, k_{j}, t_{n}\right)}{\partial x \partial t} h_{t}+\frac{1}{2} \frac{\partial^{3} f\left(x_{l}, k_{j}, t_{n}+\theta h_{t}\right)}{\partial x \partial t^{2}} h_{t}^{2} \\
& +\frac{1}{3}\left[\frac{\partial^{3} f\left(x_{l}-\theta h_{x}, k_{j}, t_{n}\right)}{\partial x^{3}}-2 \frac{\partial^{3} f\left(x_{l}-2 \theta h_{x}, k_{j}, t_{n}\right)}{\partial x^{3}}\right] h_{x}^{2},
\end{aligned}
$$

where $\theta \in(0,1)$ and it is different for all formulas. Operator $D^{-}$has the similar expansions as $D^{+}$. Supposing $g(x) \in C^{3}\left[-\frac{L}{2}, \frac{L}{2}\right]$, it is easy to derive the following conclusions by Lagrange mean value theorem,

$$
\begin{aligned}
& D^{+} g\left(x_{l}\right)=\frac{3}{2} g^{\prime}\left(x_{11}\right)-\frac{1}{2} g^{\prime}\left(x_{12}\right), \\
& \left(D^{+}\right)^{2} g\left(x_{l}\right)=\frac{5}{2} g^{\prime \prime}\left(x_{21}\right)-\frac{3}{2} g^{\prime \prime}\left(x_{22}\right), \\
& \left(D^{+}\right)^{3} g\left(x_{l}\right)=\frac{9}{2} g^{\prime \prime \prime}\left(x_{31}\right)-\frac{7}{2} g^{\prime \prime \prime}\left(x_{32}\right),
\end{aligned}
$$

where $x_{j 1}, x_{j 2} \in\left(-\frac{L}{2}, \frac{L}{2}\right)$ for $j=1,2,3$. Operator $D^{-}$satisfies the same conclusions. Assuming that $g\left(x_{l}\right)=u \exp \left(\mathrm{i} \omega l h_{x}\right)$, it is easily verified that

$$
\begin{aligned}
& D^{+} g\left(x_{l}\right)=\frac{1}{h_{x}}\left[\left(\cos \left(\omega h_{x}\right)-1\right)^{2}+\mathrm{i}\left(2-\cos \left(\omega h_{x}\right)\right) \sin \left(\omega h_{x}\right)\right] g\left(x_{l}\right), \\
& D^{-} g\left(x_{l}\right)=\frac{1}{h_{x}}\left[-\left(\cos \left(\omega h_{x}\right)-1\right)^{2}+\mathrm{i}\left(2-\cos \left(\omega h_{x}\right)\right) \sin \left(\omega h_{x}\right)\right] g\left(x_{l}\right) .
\end{aligned}
$$

Denoting $\alpha=\left(\cos \left(\omega h_{x}\right)-1\right)^{2}, \beta=\left(2-\cos \left(\omega h_{x}\right)\right) \sin \left(\omega h_{x}\right)$, we have

$$
\left(k_{j} D_{j}\right) g\left(x_{l}\right)=\left(\left|k_{j}\right| \alpha+\mathrm{i} k_{j} \beta\right) g\left(x_{l}\right)
$$

It is concluded that

$$
\left\|1+\theta k_{i} D_{j}\right\| \geq 1, \quad \theta \geq 0 .
$$

Thus it is reasonable to expand the inverse operator of $\left(I+\frac{h_{t}}{2} K D\right)$ as

$$
\left(I+\frac{h_{t}}{2} K D\right)^{-1}=I-\frac{h_{t}}{2} K D+\frac{h_{t}^{2}}{8}\left(I+\frac{\theta}{2} h_{t} K D\right)^{-3} K^{2} D^{2}
$$


where $\theta \in(0,1)$. Substituting (4.31) into operator $T$ yields

$$
\begin{aligned}
T= & \left(I+\frac{h_{t}}{2} K D\right)^{-1}\left(I-\frac{h_{t}}{2} K D-h_{t} A(x)\right) \\
= & I-h_{t} K D-h_{t} A(x)+\frac{h_{t}^{2}}{4}\left[K^{2} D^{2}+2 K D A(x)+\frac{1}{2}\left(I+\frac{\theta}{2} h_{t} K D\right)^{-3} K^{2} D^{2}\right] \\
& \quad-\frac{h_{t}^{3}}{8}\left[\left(I+\frac{\theta}{2} h_{t} K D\right)^{-3} K^{2} D^{2}\left(\frac{K D}{2}+A(x)\right)\right] .
\end{aligned}
$$

Based on the above preparations, $\Re_{3}\left(f\left(x_{l}, k_{j}, t_{n+1}\right), f\left(x_{l}, k_{j}, t_{n}\right)\right)$ can be separated into three parts

$$
\Re_{3}\left(f\left(x_{l}, k_{j}, t_{n+1}\right), f\left(x_{l}, k_{j}, t_{n}\right)\right)=: \Re_{31}+\Re_{32}+\Re_{33},
$$

where

$$
\begin{aligned}
& \Re_{31}=\frac{f\left(x_{l}, k_{j}, t_{n+1}\right)-f\left(x_{l}, k_{j}, t_{n}\right)}{h_{t}}, \\
& \Re_{32}=\frac{\hbar k_{j}}{m} D_{j}\left(\frac{f\left(x_{l}, k_{j}, t_{n+1}\right)+f\left(x_{l}, k_{j}, t_{n}\right)}{2}\right), \\
& \Re_{33}=\bar{a}_{j}(x)\left(\frac{T F\left(x_{l}, t_{n}\right)+F\left(x_{l}, t_{n}\right)}{2}\right) .
\end{aligned}
$$

According to the Taylor expansions (4.24)-(4.26), we can obtain

$$
\begin{aligned}
& \Re_{31}= \frac{\partial f\left(x_{l}, k_{j}, t_{n}\right)}{\partial t}+\frac{1}{2} \frac{\partial^{2} f\left(x_{l}, k_{j}, t_{n}\right)}{\partial t^{2}} h_{t}+\frac{1}{3 !} \frac{\partial^{3} f\left(x_{l}, k_{j}, t_{n}+\theta h_{t}\right)}{\partial t^{3}} h_{t}^{2}, \\
& \Re_{32}=\frac{\hbar k_{j}}{m} \frac{\partial f\left(x_{l}, k_{j}, t_{n}\right)}{\partial x}+\frac{\hbar k_{j}}{2 m} \frac{\partial^{2} f\left(x_{l}, k_{j}, t_{n}\right)}{\partial x \partial t} h_{t}+\frac{\hbar k_{j}}{4 m} \frac{\partial^{3} f\left(x_{l}, k_{j}, t_{n}+\theta h_{t}\right)}{\partial x \partial t^{2}} h_{t}^{2} \\
&+\frac{\hbar k_{j}}{6 m}\left[\frac{\partial^{3} f\left(x_{l} \pm \theta h_{x}, k_{j}, t_{n}\right)}{\partial x^{3}}-2 \frac{\partial^{3} f_{j}\left(x_{l} \pm 2 \theta h_{x}, t_{n}\right)}{\partial x^{3}}\right. \\
&\left.+\frac{\partial^{3} f\left(x_{l} \pm \theta h_{x}, k_{j}, t_{n+1}\right)}{\partial x^{3}}-2 \frac{\partial^{3} f\left(x_{l} \pm 2 \theta h_{x}, k_{j}, t_{n+1}\right)}{\partial x^{3}}\right] h_{x}^{2} .
\end{aligned}
$$

The error $\Re_{33}$ can be computed by (4.32)

$$
\begin{aligned}
\Re_{33}= & \bar{a}_{j}(x) \frac{(I+T) \bar{F}\left(x_{l}, t_{n}\right)}{2} \\
= & \bar{a}_{j}(x) \bar{F}\left(x_{l}, t_{n}\right)-\frac{h_{t}}{2} \bar{a}_{j}(x)\left(K \frac{\partial}{\partial x}+A(x)\right) \bar{F}\left(x_{l}, t_{n}\right) \\
& +\frac{h_{t} h_{x}^{2}}{6} \bar{a}_{j}(x) K\left[\frac{\partial^{3} \bar{F}\left(x \pm \theta h_{x}, t_{n}\right)}{\partial x^{3}}-2 \frac{\partial^{3} \bar{F}\left(x_{l} \pm 2 \theta h_{x}, t_{n}\right)}{\partial x^{3}}\right] \\
& +\frac{h_{t}^{2}}{8} \bar{a}_{j}(x)\left[K^{2} D^{2}+2 K D A(x)+\frac{1}{2}\left(I+\frac{\theta}{2} h_{t} K D\right)^{-3} K^{2} D^{2}\right] \bar{F}\left(x_{l}, t_{n}\right) \\
& -\frac{h_{t}^{3}}{16} \bar{a}_{j}(x)\left[\left(I+\frac{\theta}{2} h_{t} K D\right)^{-3} K^{2} D^{2}\left(\frac{K D}{2}+A(x)\right)\right] \bar{F}\left(x_{l}, t_{n}\right) .
\end{aligned}
$$


It is easily verified by (3.9) and (3.10) that

$$
\begin{aligned}
& \frac{\partial^{2} f\left(x, k_{j}, t\right)}{\partial t^{2}}+\frac{\hbar k_{j}}{m} \frac{\partial^{2} f\left(x, k_{j}, t\right)}{\partial x \partial t}+\bar{a}_{j}(x) \frac{\partial F(x, t)}{\partial t}=0, \\
& \frac{\partial^{2} f\left(x, k_{j}, t\right)}{\partial t^{2}}+\frac{\hbar k_{j}}{m} \frac{\partial^{2} f\left(x, k_{j}, t\right)}{\partial x \partial t}+\bar{a}_{j}(x)\left[-K \frac{\partial}{\partial x}-A\right] F(x, t)=0 .
\end{aligned}
$$

Applying (4.35)-(4.37), the expression of the truncation error $\tau_{2}$ can be calculated as

$$
\begin{aligned}
\tau_{2}=h_{t}^{2} & {\left[\frac{1}{3 !} \frac{\partial^{3} f\left(x_{n}, k_{j}, t_{n}+\theta h_{t}\right)}{\partial t^{3}}+\frac{\hbar}{m} \frac{1}{4} \frac{\partial^{3} f\left(x_{n}, k_{j}, t_{n}+\theta h_{t}\right)}{\partial x \partial t^{2}}\right.} \\
& \left.+\frac{1}{8} \bar{a}_{j}(x)\left(K^{2} D^{2}+2 K D\left(-\frac{\partial}{\partial t}-K \frac{\partial}{\partial x}\right)+\frac{1}{2}\left(I+\frac{\theta}{2} h_{t} K D\right)^{-3} K^{2} D^{2}\right) F\left(x_{l}, t_{n}\right)\right] \\
& +h_{x}^{2} \frac{\hbar k_{j}}{6 m}\left[\frac{\partial^{3} f\left(x_{l} \pm \theta h_{x}, k_{j}, t_{n}\right)}{\partial x^{3}}-2 \frac{\partial^{3} f\left(x_{l} \pm 2 \theta h_{x}, k_{j}, t_{n}\right)}{\partial x^{3}}\right. \\
& \left.+\frac{\partial^{3} f\left(x_{l} \pm \theta h_{x}, k_{j}, t_{n+1}\right)}{\partial x^{3}}-2 \frac{\partial^{3} f\left(x_{l} \pm 2 \theta h_{x}, k_{j}, t_{n+1}\right)}{\partial x^{3}}\right] \\
& +\frac{h_{t} h_{x}^{2}}{6} \bar{a}_{j}(x) K\left[\frac{\partial^{3} F\left(x \pm \theta h_{x}\right)}{\partial x^{3}}-2 \frac{\partial^{3} F\left(x_{l} \pm 2 \theta h_{x}\right)}{\partial x^{3}}\right] \\
& -\frac{h_{t}^{3}}{16} \bar{a}_{j}(x)\left[\left(I+\frac{\theta}{2} h_{t} K D\right)^{-3} K^{2} D^{2}\left(\frac{K D}{2}+A(x)\right)\right] F\left(x_{l}, t_{n}\right) .
\end{aligned}
$$

Supposing that $g(x, k, t)$ is periodic in the $k$ direction with period length $L_{k}$ and denoting

$$
\begin{aligned}
& G(x, t)=\left(g\left(x, k_{-N}, t\right), g\left(x, k_{-N+1}, t\right), \cdots, g\left(x, k_{N-1}, t\right)\right)^{\mathrm{T}}, \\
& \bar{v}_{n}=\frac{h_{k}}{L_{k}} \sum_{n=-N}^{N-1} g\left(x, k_{j}, t\right) \exp \left(\mathrm{i} \frac{2 n \pi}{L_{k}} k_{j}\right),
\end{aligned}
$$

the following estimate can be obtained

$$
\begin{aligned}
\left|\bar{a}_{j}(x) G(x, t)\right| & =\left|\frac{\mathrm{i}}{\hbar} \sum_{-N}^{N-1} \bar{v}_{n} V_{d}\left(x, \frac{2 n \pi}{L_{k}}\right) \exp \left(\mathrm{i} \frac{2 n \pi}{L_{k}} k_{j}\right)\right| \\
& \leq \frac{2 M}{\hbar} \sum_{-N}^{N-1}\left|\bar{v}_{n}\right| .
\end{aligned}
$$

According to (4.15), $\left|\bar{a}_{j}(x) G(x, t)\right|$ is bounded as the function $g(x, k, t)$ satisfies

$$
\left|\frac{\partial^{2} g(x, k, t)}{\partial k^{2}}\right| \leq C,
$$

where $C$ is a constant. From (4.27), we can get

$$
\begin{aligned}
& k_{j}^{2} D_{j}^{2} f\left(x_{l}, k_{j}, t_{n}\right)=\frac{5}{2} k_{j}^{2} \frac{\partial^{2} f\left(x_{11}, k_{j}, t\right)}{\partial x^{2}}-\frac{3}{2} k_{j}^{2} \frac{\partial^{2} f\left(x_{12}, k_{j}, t\right)}{\partial x^{2}}, \\
& k_{j} D_{j} \frac{\partial f\left(x_{l}, k_{j}, t_{n}\right)}{\partial t}=\frac{3}{2} k_{j} \frac{\partial^{2} f\left(x_{21}, k_{j}, t_{n}\right)}{\partial x \partial t}-\frac{1}{2} k_{j} \frac{\partial^{2} f\left(x_{22}, k_{j}, t_{n}\right)}{\partial x \partial t}, \\
& k_{j}^{2} D_{j} \frac{\partial f\left(x_{l}, k_{j}, t_{n}\right)}{\partial x}=\frac{3}{2} k_{j}^{2} \frac{\partial^{2} f\left(x_{31}, k_{j}, t\right)}{\partial x^{2}}-\frac{1}{2} k_{j}^{2} \frac{\partial^{2} f\left(x_{32}, k_{j}, t\right)}{\partial x^{2}} .
\end{aligned}
$$


Applying (4.38), (4.41), (4.42) and assumption (4.22), the estimation (4.23) for error $\tau_{2}$ is arrived and $\tau_{2}$ has the second-order accuracy with respect $h_{x}$ and $h_{t}$.

The estimation of local truncation error $\tau\left(x_{l}, k_{j}, t_{n}\right)$ is summarized in the following theorem.

Theorem 4.1. Under the Assumption of Lemma 1 and Lemma 2, the local truncation error $\tau\left(x_{l}, k_{j}, t_{n}\right)$ defined in (4.1) satisfies

$$
\left|\tau\left(x_{l}, k_{j}, t_{n}\right)\right|=C_{1} h_{t}^{2}+C_{2} h_{x}^{2}+C_{3} h_{k}^{p-1},
$$

where

$$
\begin{aligned}
& C_{1}=\left(\frac{1}{6}+\frac{\hbar}{4 m}+\frac{28 M}{\hbar}\left(2+\frac{L_{k}}{2}\right)^{2}\left(1+\left(\frac{L_{k}}{\pi}\right)^{2}\right)\right) P, \\
& C_{2}=\left(\frac{L_{k} \hbar}{2 m}\right) P, \quad C_{3}=\frac{M Q L_{k}}{2 \hbar \pi^{p+1}}\left(\frac{5 p-1}{p-1}\right),
\end{aligned}
$$

and $C_{i}(i=1,2,3)$ are constants which are independent of $h_{x}, h_{t}$ and $h_{k}$.

\subsection{Stability}

The EI scheme (3.16) can be reformulated in matrix form

$$
\left\{\begin{array}{l}
\frac{\bar{F}_{l}^{n+1}-F_{l}^{n}}{h_{t}}+K D\left(\frac{F_{l}^{n}+\bar{F}_{l}^{n+1}}{2}\right)+A_{l} F_{l}^{n}=0 \\
\frac{F_{l}^{n+1}-F_{l}^{n}}{h_{t}}+K D\left(\frac{F_{l}^{n}+F_{l}^{n+1}}{2}\right)+A_{l}\left(\frac{F_{l}^{n}+\bar{F}_{l}^{n+1}}{2}\right)=0 .
\end{array}\right.
$$

The von Neumann method is used to analyze the stability of the EI scheme through (4.45). Supposing that $F_{l}^{n}=U^{n} e^{\mathrm{i} l \omega h_{x}}$ and vector $U^{n}=\left(u_{-N}^{n}, u_{-N+1}^{n}, \cdots, u_{N-1}^{n}\right)^{\mathrm{T}}$, we insert the expressions into (4.45). It is easily verified that

$$
D^{+} F_{l}^{n}=\frac{1}{h_{x}}(\alpha+\mathrm{i} \beta) F_{l}^{n}, \quad D^{-} F_{l}^{n}=\frac{1}{h_{x}}(-\alpha+\mathrm{i} \beta) F_{l}^{n},
$$

where $\alpha=\left(\cos \left(\omega h_{x}\right)-1\right)^{2}, \beta=\left(2-\cos \left(\omega h_{x}\right)\right) \sin \left(\omega h_{x}\right)$. Denoting $\lambda=\frac{\hbar h_{t}}{2 m h_{x}}$ and fixing the matrix $A_{l}$ as $A$, we can derive that

$$
\left\{\begin{array}{l}
(I+\lambda \alpha|K|+\mathrm{i} \lambda \beta K) \bar{U}^{n+1}=(I-\lambda \alpha|K|+\mathrm{i} \lambda \beta K) U^{n}-h_{t} A U^{n}, \\
(I+\lambda \alpha|K|+\mathrm{i} \lambda \beta K) U^{n+1}=(I-\lambda \alpha|K|+\mathrm{i} \lambda \beta K) U^{n}-\frac{h_{t}}{2} A U^{n}-\frac{h_{t}}{2} A \bar{U}^{n+1},
\end{array}\right.
$$

where $|K|=\operatorname{diag}\left(\left|k_{-N}\right|,\left|k_{-N+1}\right|, \ldots,\left|k_{N-1}\right|\right)$ and $K=\operatorname{diag}\left(k_{-N}, k_{-N+1}, \ldots, k_{N-1}\right)$. Introducing the following notations

$$
\mathrm{H}_{1}=I+\alpha \lambda|K|+\mathrm{i} \beta \lambda K, \quad \mathrm{H}_{2}=I-\alpha \lambda|K|+\mathrm{i} \beta \lambda K,
$$

substituting these into (4.47) yields

$$
\mathrm{U}^{n+1}=\mathrm{H}_{1}^{-1} \mathrm{H}_{2} \mathrm{U}^{n}-\frac{h_{t}}{2} \mathrm{H}_{1}^{-1} A \mathrm{U}^{n}-\frac{h_{t}}{2} \mathrm{H}_{1}^{-1} A \mathrm{H}_{1}^{-1}\left(\mathrm{H}_{2}-h_{t} A\right) \mathrm{U}^{n} .
$$


Matrix $\mathrm{H}_{1}^{-1}$ is a diagonal matrix with the $j$-th diagonal element $h_{j}$

$$
h_{j}=\frac{1}{1+\alpha \lambda\left|k_{j}\right|+\mathrm{i} \beta \lambda k_{j}} .
$$

$\mathrm{H}_{1}{ }^{-1} \mathrm{H}_{2}$ is also a diagonal matrix with the $j$-th diagonal element $g_{j}$

$$
g_{j}=\frac{1-\alpha \lambda\left|k_{j}\right|+\mathrm{i} \beta \lambda k_{j}}{1+\alpha \lambda\left|k_{j}\right|+\mathrm{i} \beta \lambda k_{j}} .
$$

Observe in (4.49) and (4.50) that $\left|g_{j}\right| \leq 1$ and $\left|h_{j}\right| \leq 1$ for $\alpha \geq 0$. Thus we have

$$
\left\|\mathrm{U}^{n+1}\right\|_{2} \leq\left\|\mathrm{U}^{n}\right\|_{2}+h_{t}\|A\|_{2}\left\|\mathrm{U}^{n}\right\|_{2}+\frac{h_{t}^{2}}{2}\|A\|_{2}^{2}\left\|\mathrm{U}^{n}\right\|_{2} \text {. }
$$

It is obvious that the matrix $2 \pi \hbar \cdot A$ is the discretization of the pseudo-differential term defined in $(3.11)$.

Lemma 4.3. Under the assumptions of Theorem 4.1, let the $2 N \times 2 N$ matrix $A$ with the elements $(A(x))_{j j^{\prime}}=a_{j j^{\prime}}$ be defined in (3.11). Assuming $|V(x)| \leq M$, then

$$
\|A(x)\|_{2} \leq \frac{2}{\hbar} M
$$

holds.

Proof. The $j$-th element of $A F$ can be written as

$$
\begin{aligned}
(A F)_{j} & =\frac{h_{k}}{2 \pi \hbar} \sum_{j^{\prime}=-N}^{N-1} \widetilde{V}_{w}\left(x, k_{j}-k_{j^{\prime}}\right) f\left(x, k_{j^{\prime}}, t\right) \\
& =\frac{\mathrm{i}}{\hbar} \frac{1}{2 N} \sum_{j^{\prime}=-N}^{N-1} \sum_{n=-N}^{N-1} V_{d}\left(x, n h_{r}\right) \exp \left(-\mathrm{i} n h_{r}\left(k_{j}-k_{j^{\prime}}\right)\right) f\left(x, k_{j^{\prime}}, t\right) .
\end{aligned}
$$

One can find that $A F$ is a discrete convolution, i.e.,

$$
A F=\frac{\mathrm{i}}{\hbar} \bar{V}_{d} * F, \quad \bar{V}_{d}=\left\{\frac{1}{2 N} \sum_{n=-N}^{N-1} V_{d}\left(x, n h_{r}\right) \exp \left(-\mathrm{i} n h_{r} k_{j}\right)\right\}_{j=-N}^{N-1},
$$

where $\bar{V}_{d}$ is periodic with the period length $2 N$ and $F=F\left(x_{l}, t_{n}\right)$. Applying Parseval equation to the discrete convolution yields

$$
\|A F\|_{2}=\frac{1}{\hbar}\left\|\bar{V}_{d} * F\right\|_{2}=\frac{1}{\hbar}\left\|V_{d}\left(x, n h_{r}\right) \widehat{F}\right\|_{2} \leq \frac{1}{\hbar}\left\|V_{d}\left(x, n h_{r}\right)\right\|_{\infty}\|F\|_{2},
$$

where $\widehat{F}$ is the Fourier transform of $F$. On the other hand, we have

$$
\left\|V_{d}\left(x, n h_{r}\right)\right\|_{\infty} \leq 2\|V(x)\|_{\infty}=2 M .
$$

Using (4.55) and (4.56), (4.52), we can arrive the conclusion.

Denoting $M_{1}=\frac{2}{\hbar} M$, it is derived that

$$
\left\|\mathrm{U}^{n+1}\right\|_{2} \leq\left(1+h_{t} M_{1}+\frac{1}{2} h_{t}^{2} M_{1}^{2}\right)\left\|\mathrm{U}^{n}\right\|_{2} .
$$

According to von Neumann theorem, the conclusion can be obtained as follow. 
Theorem 4.2. Under the assumptions of Theorem 4.1, there exists a constant $M_{1}=\frac{2}{\hbar} M$ such that $\|A(x)\|_{2} \leq M_{1}$. Then the EI scheme (4.45) is stable

$$
\left\|F^{n+1}\right\|_{2}^{h} \leq\left(1+h_{t} M_{1}+\frac{1}{2} h_{t}^{2} M_{1}^{2}\right)\left\|F^{n}\right\|_{2}^{h},
$$

where norm $\|\cdot\|_{2}^{h}$ is defined by $\left\|F^{n}\right\|_{2}^{h}=\sqrt{\sum_{l=1}^{N_{x}-1} \sum_{j=-N}^{N-1}\left|f_{l j}^{n}\right|^{2} h_{k} h_{x}}$.

\subsection{Convergence}

In this section, we describe the convergence theorem of the hybrid EI scheme. Firstly, we rewrite (4.45) as

$$
\left(I+\frac{h_{t}}{2} K D\right) F_{l}^{n+1}=\left(I-\frac{h_{t}}{2} K D+\frac{h_{t}}{2} A_{l}(I+T)\right) F_{l}^{n} .
$$

Denoting

$$
L=I+\frac{h_{t}}{2} K D, \quad R=I-\frac{h_{t}}{2} K D+\frac{h_{t}}{2} A_{l}(I+T),
$$

it is obvious that $\left\|L^{-1}\right\| \leq 1$ according to (4.30). Numerical solution $F_{l}^{n}$ and exact solution $F\left(x_{l}, t_{n}\right)$ satisfy

$$
L F_{l}^{n+1}-R F_{l}^{n}=0, \quad L F\left(x_{l}, t_{n+1}\right)-R F\left(x_{l}, t_{n}\right)=h_{t} \Re_{3} F\left(x_{l}, t_{n}\right) .
$$

According to the stability conclusion (4.58), we can get

$$
\left\|F^{n+1}\right\|_{2}^{h}=\left\|L^{-1} R F^{n}\right\|_{2}^{h} \leq\left(1+h_{t} M_{1}+\frac{1}{2} h_{t}^{2} M_{1}^{2}\right)\left\|F^{n}\right\|_{2}^{h} .
$$

The error vector between numerical solution $F_{l}^{n}$ and exact value $F\left(x_{l}, t_{n}\right)$ is denoted by

$$
E\left(x_{l}, t_{n}\right)=F\left(x_{l}, t_{n}\right)-F_{l}^{n} .
$$

Due to (4.61), the error vector $E\left(x_{l}, t_{n}\right)$ satisfies

$$
E\left(x_{l}, t_{n+1}\right)=L^{-1} R E\left(x_{l}, t_{n}\right)+h_{t} L^{-1} \Re_{3} F\left(x_{l}, t_{n}\right) .
$$

Applying the conclusions in Theorem 4.2 and (4.62), it is derived that

$$
\left\|E^{n+1}\right\|_{2}^{h} \leq\left(1+M_{1} h_{t}+\frac{1}{2} M_{1}^{2} h_{t}^{2}\right)\left\|E^{n}\right\|_{2}^{h}+h_{t} L_{x} L_{k} \tau_{\infty}
$$

where $\tau_{\infty}=C_{1} h_{t}^{2}+C_{2} h_{x}^{2}+C_{3} h_{k}^{p-1}$ and $\left\|\mathrm{E}^{n}\right\|_{2}^{h}=\sqrt{\sum_{l=1}^{N_{x}-1} \sum_{j=-N}^{N-1}\left|f_{l j}^{n}-f\left(x_{l}, k_{j}, t_{n}\right)\right|^{2} h_{k} h_{x}}$. It is a trivial process to arrive at the convergence conclusion.

Theorem 4.3. Under the assumptions of Theorems 4.1 and 4.2 , the global error $E^{n}$ defined in (4.63) satisfies

$$
\left\|E^{n}\right\|_{2}^{h} \leq e^{M_{1} T}\left\|E^{0}\right\|_{2}^{h}+\frac{L_{x} L_{k}\left(e^{M_{1} T}-1\right)}{M}\left(C_{1} h_{t}^{2}+C_{2} h_{x}^{2}+C_{3} h_{k}^{p-1}\right)
$$

for all $x \in\left[-\frac{L}{2}, \frac{L}{2}\right], k \in\left[-\frac{L_{k}}{2}, \frac{L_{k}}{2}\right]$ and $0 \leq t \leq T$, where $C_{j},(j=1,2,3)$ and $M_{1}$ are constants which are independent of $h_{t}, h_{x}$ and $h_{k}$. 


\section{Numerical Results}

In this section, we carry out a series of numerical experiments to illustrate the convergence of the EI scheme. The tunneling of a Gaussian wave packet through a potential barrier is simulated. The initial distribution is set as a normalized Gaussian function

$$
f(x, k, 0)=\frac{2}{\pi} \exp \left(-\frac{(x-0.5)^{2}}{0.5}\right) \exp \left(-\frac{(k+0.25)^{2}}{0.5}\right),
$$

which is located to the right of a potential barrier. The potential is chosen to be a Gaussian function of the form

$$
V(x)=v_{0} \exp \left(-\frac{x^{2}}{\sigma^{2}}\right),
$$

where parameter $v_{0}$ denotes the height of potential barrier and parameter $\sigma$ indicates the width of potential barrier. Some variables, parameters and their values or units in (2.1) and (2.3) used in our codes are listed in Table 5.1.

Table 5.1: The meaning, units and values of the independent variables and coefficients.

\begin{tabular}{|lccc|}
\hline Variable & Meaning & Unit & Value \\
\hline$x$ & position & $\mathrm{nm}$ & \\
$k$ & wave number & $\mathrm{nm}^{-1}$ & \\
$t$ & time & $10^{-15} \mathrm{~s}$ & \\
$m$ & effective mass & $\mathrm{kg}$ & $0.61 \times 10^{-31}$ \\
$\hbar$ & reduced Plank constant & $\mathrm{J.s}$ & $1.055 \times 10^{-34}$ \\
$V(x)$ & potential & $\mathrm{eV}$ & \\
\hline
\end{tabular}

The density function is the zero momentum of the Wigner function

$$
n(x, t)=\int_{-\infty}^{\infty} f(x, k, t) \mathrm{d} k
$$

which can be calculated by

$$
n\left(x_{l}, t_{n}\right)=\sum_{j=-N}^{N-1} f_{l j}^{n} h_{k}
$$

\subsection{The convergence order of the EI scheme}

Firstly, we implement our method for the Wigner equation with zero potential, i.e., $v_{0}=0$. In this special case, the Wigner equation is reduced into a set of advection equations

$$
\frac{\partial f(x, k, t)}{\partial t}+\frac{k \hbar}{m} \frac{\partial f(x, k, t)}{\partial x}=0
$$

The exact solution of (5.5) and (5.1) is

$$
f(x, k, t)=\frac{2}{\pi} \exp \left(-\frac{\left(x-0.5-\frac{k \hbar}{m} t\right)^{2}}{0.5}\right) \exp \left(-\frac{(k+0.25)^{2}}{0.5}\right) .
$$

The second-order upwind scheme is used to discretize the advection term and the explicitimplicit scheme is used to discretize time integration. The simulation region is chosen as 
Table 5.2: The numerical error and numerical order of accuracy at different spatial mesh size $h_{x}$ for the Wigner equation with potential $v_{0}=0$.

\begin{tabular}{|ccccccc|}
\hline$h_{x}$ & 0.5 & 0.25 & 0.125 & 0.0625 & 0.03125 & 0.0156 \\
$\mathrm{E}$ & 0.0808 & 0.0315 & 0.0092 & 0.0024 & $6.0121 \mathrm{e}-004$ & $1.5029 \mathrm{e}-004$ \\
$p$ & $*$ & 1.3590 & 1.7756 & 1.9386 & 1.9971 & 2.0001 \\
\hline
\end{tabular}

$L=6, L_{t}=0.4$, which is large enough to reasonably impose homogenous Dirichlet boundary conditions. The numerical error in $L^{2}$-norm is defined by

$$
\mathrm{E}(h)=\left[h_{x} h_{k} \sum_{l} \sum_{j}\left(f_{l j}^{n}(h)-f\left(x_{l}, k_{j}, t_{n}\right)\right)^{2}\right]^{\frac{1}{2}},
$$

where $f\left(x_{l}, k_{j}, t_{n}\right)$ denotes the exact value at $\left(x_{l}, k_{j}, t_{n}\right)$ and $f_{l j}^{n}(h)$ denotes the numerical value computed with mesh step $h$. If the exact solution of the Wigner equation can not be obtained as the reference solution, we can estimate the error on each grid by using the next finer grid as the reference solution [24]. In this case, the numerical error in $L^{2}$-norm is defined by the difference between two solutions with refined steps as

$$
\mathrm{E}(h)=\left[h_{x} h_{k} \sum_{l} \sum_{j}\left(f_{l j}^{n}(h)-f_{l j}^{n}\left(\frac{h}{2}\right)\right)^{2}\right]^{\frac{1}{2}} .
$$

The convergence order of the numerical solution with respect to $h$ is defined as

$$
p=\log _{2} \frac{\mathrm{E}(h)}{\mathrm{E}\left(\frac{h}{2}\right)} .
$$

In order to analyze the convergence order of the EI scheme with respect to $h_{x}$, the time step $h_{t}$ is set to be 0.001 which is too small to affect the numerical solution.

When the mesh size $h_{x}$ is halved every time, the numerical error and the convergence order are calculated and listed in Table 5.2. It shows the convergence order with respect to $h_{x}$ is

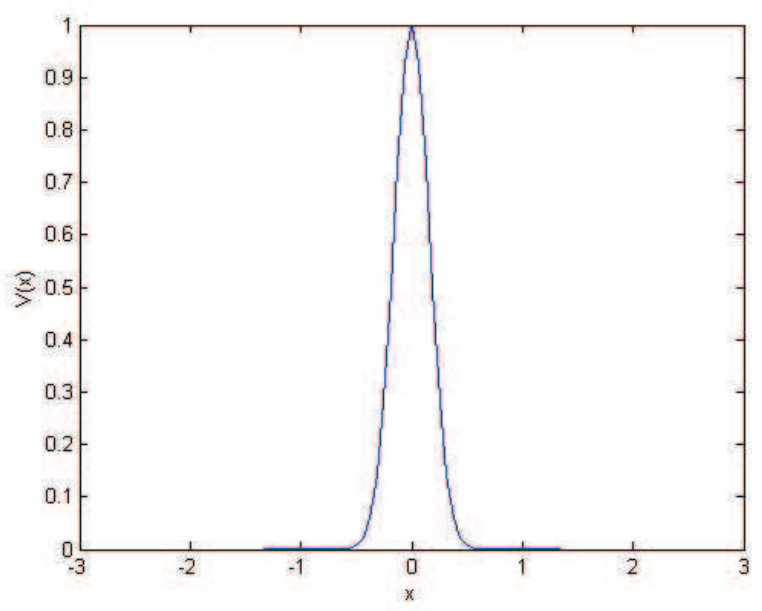

Fig. 5.1. The potential function $V(x)$, where $v_{0}=1$ and $\sigma^{2}=0.05$. 
approaching to 2 and this verifies the results of convergence analysis. The convergence orders with respect to $h_{t}$ and $h_{k}$ are also investigated by simulating a Gaussian wave packet function tunneling through a time-independent potential barrier with barrier height $v_{0} \neq 0$. Firstly, we investigate the convergence of the density function with respect to $h_{k}$ and $L_{k}$. In the proposed hybrid EI scheme, the parameters $L_{k}$ and $h_{k}$ are related to $h_{r}$ and $L_{r}$ by $L_{k}=\frac{2 \pi}{h_{r}}, h_{k}=\frac{2 \pi}{L_{r}}$. Figure 5.2(a) shows the convergence of the density function $n(x, t)$ with respect to $h_{r}$ or the length of the wave number domain $L_{k}$. It is found out that $h_{r}=\frac{\pi}{4}\left(\mathrm{eg}, L_{k}=8.0\right)$ is good enough for the density convergence. Figure 5.2(b) presents that the density function $n(x, t)$ converges with the coherence length $L_{r}$. The numerical test illustrates that it is not necessary to use too big coherence length $L_{r}$ or too small wave number step $h_{k}$. It is demonstrated that the hybrid EI scheme has spectral accuracy with respect to $h_{k}$ in Table 5.3. Table 5.4 studies the convergence order with respect to $h_{t}$ with fixing $h_{x}=0.1, h_{k}=0.1$ and $L_{k}=8.0$. It shows the hybrid EI scheme has the second-order accuracy with respect to the time step $h_{t}$.

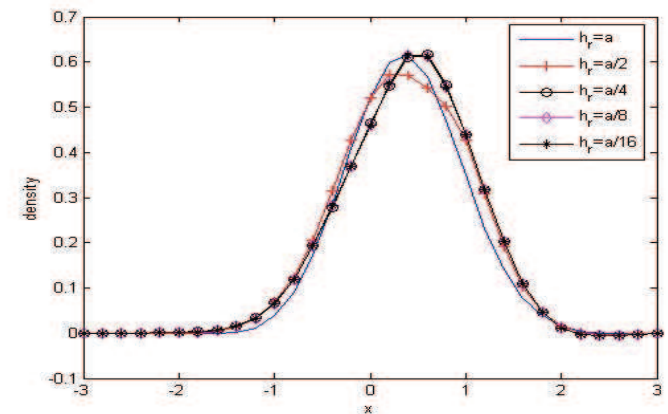

(a) The convergence of the density function with respect to $h_{r}$, where $h_{k}=0.1$ and $a=\pi$.

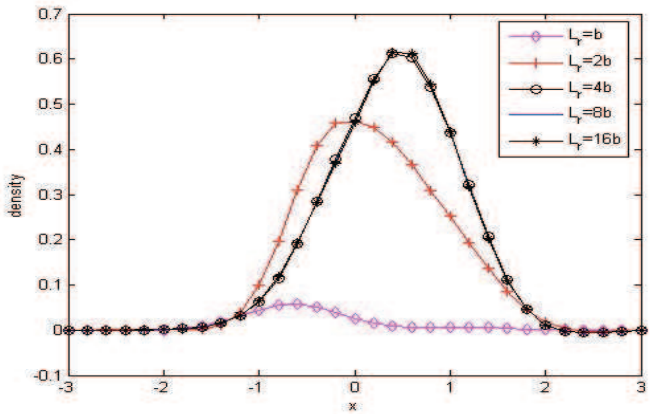

(b) The convergence of the density function with respect to $L_{r}$, where $h_{r}=0.1$ and $b=2$.

Fig. 5.2. Density functions calculated by using the EI scheme with different $h_{r}$ and $L_{r}$.

Table 5.3: The numerical error and the numerical accuracy of order with respect to $h_{k}$ when applying the EI scheme for the Wigner equation with a Gaussian potential barrier.

\begin{tabular}{|lccccc|}
\hline $2 N$ & 20 & 40 & 80 & 160 & 320 \\
$\mathrm{E}$ & $*$ & 0.0879 & 0.0345 & 0.0017 & $1.5750 \mathrm{e}-005$ \\
$p$ & $*$ & $*$ & 1.3474 & 4.3735 & 6.7253 \\
\hline
\end{tabular}

Table 5.4: The numerical error and the numerical accuracy of order with respect to $h_{t}$ when applying the EI scheme for the Wigner equation with a Gaussian potential barrier.

\begin{tabular}{|cccccc|}
\hline$h_{t}$ & 0.1 & 0.05 & 0.025 & 0.0125 & 0.00625 \\
$\mathrm{E}$ & $*$ & 0.0110 & 0.0047 & 0.0014 & $1.3259 \mathrm{e}-005$ \\
$p$ & $*$ & $*$ & 1.2268 & 1.7472 & 1.9429 \\
\hline
\end{tabular}

The evolution of the Gaussian wave packet is depicted in Figure 5.3. The initial Gaussian distribution function (5.1) is given in Figure 5.3(a). Figure 5.3(b) shows the distribution function $f(x, k, t)$ at $T=0.4$ with barrier height $v_{0}=0.0$ and presents the smooth dispersion of 


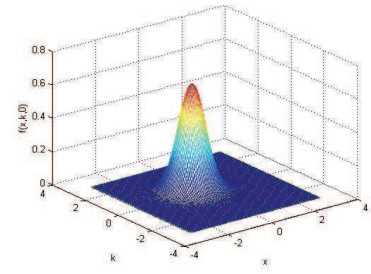

(a) $T=0$

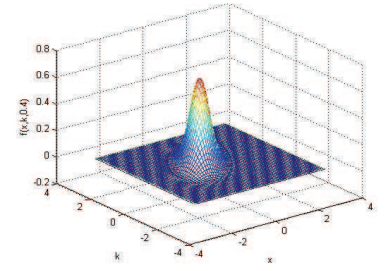

(b) $T=0.4, v_{0}=0, \sigma^{2}=0.05$

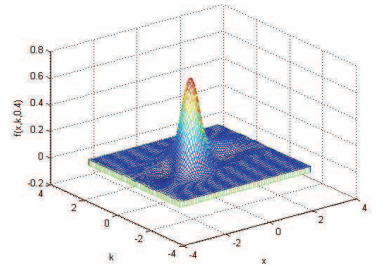

(c) $T=0.4, v_{0}=1, \sigma^{2}=0.05$

Fig. 5.3. The evolution of the Gaussian wave packet.

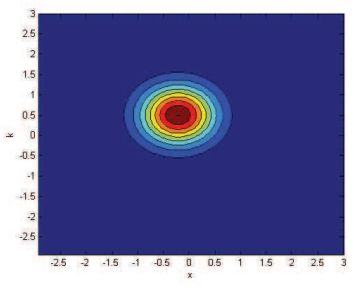

(a) $T=0$

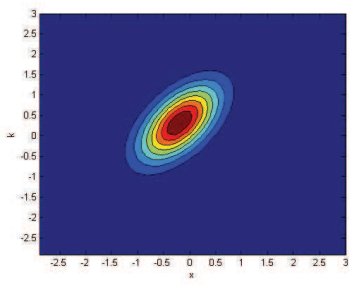

(b) $T=0.4, v_{0}=0, \sigma^{2}=0.05$

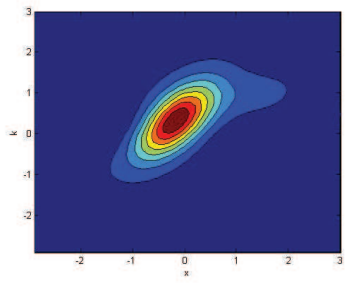

(c) $T=0.4, v_{0}=1, \sigma^{2}=0.05$

Fig. 5.4. The contour lines of the Gaussian wave packet.
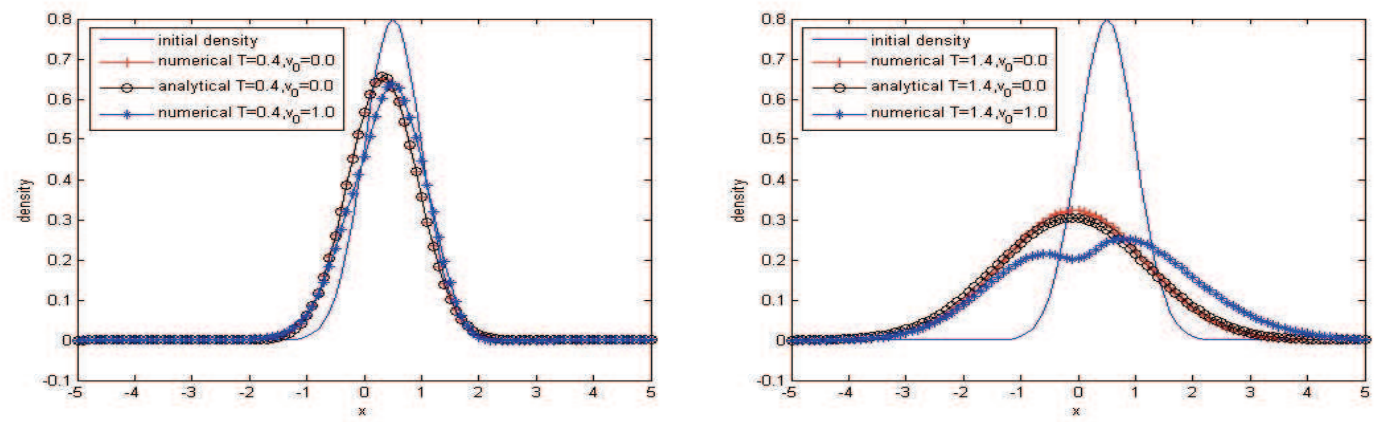

Fig. 5.5. Density functions calculated by using the EI scheme at $T=0.4$ and $T=1.4$, where $\sigma^{2}=0.05$.

a free Gaussian wave packet; Figure 5.3(c) depicts the tunneling of the Gaussian wave packet through a Gaussian potential barrier with barrier height $v_{0}=1.0$. Negative values of the Wigner distribution appearing in Figure 5.3(c) reflect the quantum property of the Wigner equation. In order to observe the scattering effects of the potential barrier clearly, the corresponding contours are plotted in Figure 5.4. Obviously, the free Gaussian wave packet disperses smoothly while the Gaussian wave packet tunneling through a barrier oscillates due to the scattering effect of the potential barrier.

\subsection{The scattering effect}

The scattering effect can be investigated clearly by computing the density function (5.4). It is obvious that the initial density function is a Gaussian function with respect to $x$ according to the initial Gaussian wave packet (5.1). The density functions at $T=0.4$ and $T=1.4$ are 


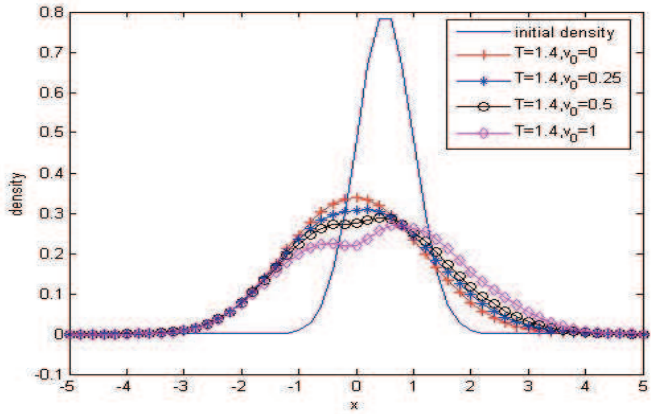

(a) $\sigma^{2}=0.05$

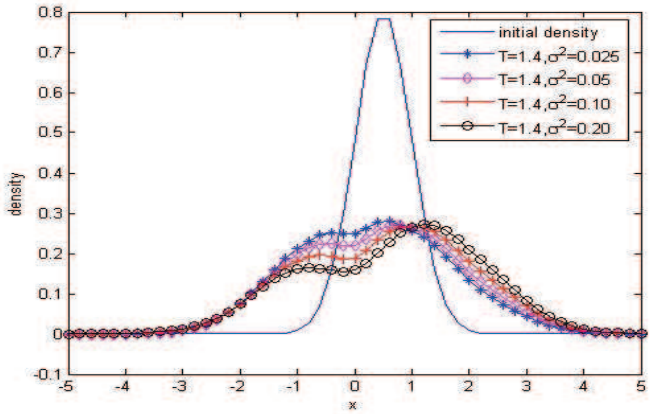

(b) $v_{0}=1$

Fig. 5.6. Density functions with different barrier height and width calculated by using the EI scheme.

depicted in Figure 5.5. The left figure is the density at $T=0.4$ with $v_{0}=0.0$ and $v_{0}=1.0$. It shows the density functions disperse and shift to the left. The density function with $v_{0}=1.0$ moves a little slowly than the density function of the free Gaussian packet. The simulation time scale is enlarged to $T=1.4$ in the right figure. It shows that the density function with $v_{0}=1.0$ collapses at $x=0$ where the barrier center is located for the scattering effect of the barrier. The effects of the barrier height and width to the scattering phenomenon are studied in Figure 5.6. The left figure presents that the density functions collapse more seriously as the barrier height increases. It indicates that a larger portion of the wave is reflected back when encountering a higher barrier. The right figure gives the density functions with different values of $\sigma$ which indicates the width of the barrier. It can be easily seen that the density functions collapse less as the width is decreased. It shows a larger portion of the wave is reflected back when encountering a thicker barrier.

\section{Conclusion}

In this paper, we proposed a hybrid explicit-implicit scheme that combines the finite difference method and a spectral method for the time-dependent Wigner equation. The consistence error and the stability of the full discretization are analyzed. In the numerical results, we demonstrate that the scheme is convergent second-order accuracy with respect to time and space variable and the spectral accuracy with respect to $h_{k}$. The tunneling of a Gaussian wave packet through a Gaussian potential barrier is simulated, and the effects of the barrier's shape to the scattering phenomena are investigated. It is concluded that a larger portion of the wave is reflected when the height and width of the barrier increase.

Acknowledgments. This research was supported in part by the NSFC (91434201, 91630130, 11671038, 11421101).

\section{References}

[1] E. Wigner, On the quantum correction for thermodynamic equilibrium, Phys. Rev., 40:5 (1932), 749-759.

[2] D. Ferry and S. Goodnick, Transport in Nanostructures, Cambridge Univ. Press, Cambridge, U.K, 1997. 
[3] J. Shi and I. Gamba, A high order local solver for Wigner equation, International Workshop on Computational Electronics, (2004), 245-246.

[4] W. Schleich, Quantum Optics in Phase Space, Wiley, England, 2001.

[5] W. Frensley,Wigner function model of a resonant-tunneling semiconductor device, Phys. Rev. B, 36 (1987), 1570-1580.

[6] K. Jensen and F. Buot, Numerical simulation of transient response and resonant tunneling characteristics of double barrier semiconductor structures as a function of experimental parameters, J. Appl. Phys., 65:12 (1989), 5248-5250.

[7] K. Jensen and F. Buot, Numerical aspects on the simulation of IV characteristics and switching times of resonant tunneling diodes, J. Appl. Phys., 67 (1990), 2153-2155.

[8] A. Dorda and F. Schürrer, A WENO-solver combined with adaptive momentum discretization for the Wigner transport equation and its application to resonant tunneling diodes, J. Comput. Phys., 284 (2015), 95-116.

[9] C. Ringhofer, A spectral method for the numerical solution of quantum tunneling phenomena, SIAM J. Num. Anal., 27 (1990), 32-50.

[10] C. Ringhofer, A spectral collocation technique for the solution of the Wigner-Poisson problem, SIAM Journal on Numerical Analysis, 29:3 (1992), 679-700.

[11] K. Kim and B. Lee, On the high order numerical calculation schemes for the Wigner transport equation, Solid-State Electronics, 43:12 (1999), 2243-2245.

[12] D. Yin, M. Tang, and S. Jin, The Gaussian beam method for the Wigner equation with discontinuous potentials, Inverse Problems \& Imaging, 7:3 (2013).

[13] Z. Cai, Y. Fan, R. Li, T. Lu, and Y. Wang, Quantum hydrodynamics models by moment closure of Wigner equation, J. Math. Phys., 53:103503 (2012).

[14] R. Li, T. Lu, Y. Wang, and W. Yao, Numerical method for high order hyperbolic moment system of Wigner equation, Commun. Comput. Phys., 9:3 (2014), 659-698.

[15] S. Shao, T. Lu, and W. Cai, Adaptive conservative cell average spectral element methods for transient Wigner equation in quantum transport, Commun. Comput. Phys., 9 (2011), 711-739.

[16] P. Markowich and C. Ringhofer, An analysis of the quantum liouville equation, Z. angew. Math. Mech., 69 (1989), 121-127.

[17] H. Jiang and W. Cai, Effect of boundary treatments on quantum transport current in the Green's function and Wigner distribution methods for a nano-scale DG-MOSFET, J. Comput. Phys., 229:12 (2010), 4461-4475.

[18] H. Jiang, W. Cai, and R. Tsu, Accuracy of the frensley inflow boundary condition for Wigner equations in simulating resonant tunneling diodes, J. Comput. Phys., 230 (2011), 2031-2044.

[19] H. Jiang, T. Lu, and W. Cai, A device adaptive inflow boundary condition for Wigner equations of quantum transport, J. Comput. Phys., 248 (2014), 773-786.

[20] A. Arnold, H. Lange, and P. Zweifel, A discrete-velocity, stationary Wigner equation, J. Math. Phys., 41:11 (2000), 7167-7180.

[21] T. Goudon, Analysis of a semidiscrete version of the Wigner equation, SIAM J. Numerical Analysis, 40:6 (2003), 2007-2025.

[22] R. Li, T. Lu, and Z.-P. Sun, Stationary wigner equation with inflow boundary conditions: Will a symmetric potential yield a symmetric solution?, SIAM J. Appl. Math., 70:3 (2014), 885-897.

[23] R. Li, T. Lu, and Z.-P. Sun, Parity-decomposition and moment analysis for stationary Wigner equation with inflow boundary conditions, Frontiers of Mathematics in China, 12:4 (2017), 907919.

[24] R. LeVeque, Finite Difference Methods for Ordinary and Partial Differential Equations: SteadyState and Time-Dependent Problems, Society for Industrial and Applied Mathematics, Philadelphia, (2007). 